\title{
Mucous Polyp of Cervix
}

National Cancer Institute

\section{Source}

National Cancer Institute. Mucous Polyp of Cervix. NCI Thesaurus. Code C34826.

A polypoid lesion that arises from the cervix and contains mucus. 